\title{
The Value of Sonography in Distinguishing Follicular Thyroid Carcinoma from Adenoma
}

\author{
Wen $\mathrm{Li}\left(\mathrm{ID}^{1,2}\right.$ \\ Qing Song ${ }^{2}$ \\ Yu Lan (iD) ${ }^{2}$ \\ Jie $\mathrm{Li}^{3}$ \\ Ying Zhang $\mathbb{D D}^{2}$ \\ Lin Yan (D) ${ }^{2}$ \\ Yingying $\mathrm{Li}^{2}$ \\ Yan Zhang iD ${ }^{2}$ \\ Yukun Luo iD ${ }^{2}$ \\ 'Department of Ultrasound, Medical \\ School of Chinese PLA, Beijing, People's \\ Republic of China; ${ }^{2}$ Department of \\ Ultrasound, The First Medical Center \\ Chinese PLA General Hospital, Beijing, \\ People's Republic of China; ${ }^{3}$ Department \\ of Pathology, The First Medical Center, \\ Chinese PLA General Hospital, Beijing, \\ People's Republic of China
}

Correspondence: Yukun Luo; Yan Zhang No. 28 Fuxing Road, Haidian District, Beijing, 100853, People's Republic of China

Tel +86I33II373556; +86I358|93750 I

Fax +8601055499255

Email luoyk30I@I26.com;

zhangyaner30I@I26.com
Purpose: Differentiation between follicular thyroid carcinomas (FTCs) and follicular thyroid adenomas (FTAs) is difficult and the sonographic features of FTC are not yet fully established. The purpose of this study is to explore the sonographic features of FTC and the value of sonography in differentiating FTCs from FTAs.

Patients and Methods: A total of 28 pathologically proven FTCs and 53 FTAs in 78 patients who were performed thyroid surgery were included in this retrospective study. The sonographic features of each tumor including an interrupted halo, satellite nodule(s) with or without halo ring, local irregularity of margin and cluster of grapes sign were evaluated. A mode image of FTC halo was built up in our study. The frequencies of the sonographic features were compared by chi-square test or Fisher exact test between FTCs and FTAs. The relative risk of malignancy was assessed by logistic regression analysis.

Results: Logistic regression analysis showed that a thick, irregular and/or interrupted halo with or without satellite nodule(s), hypoechoic or marked hypoechoic echogenicity, a predominantly solid pattern, cluster of grapes sign, micro-or macro-calcifications, rim calcifications correlated with significant increases in relative risk for FTCs (odds ratio 11.48 (1.37-96.56), 6.74 (1.05-43.30), 17.51 (1.78-172.53), 9.55 (1.44-63.46), 9.36 (1.25-$70.15)$ and 17.45 (1.04-292.65), respectively, $\mathrm{p}<0.05)$. Two new sonographic features, an interrupted halo and satellite nodule(s) with or without halo ring, can only be found in FTCs. Conclusion: An interrupted halo and satellite nodule(s) with or without halo ring are specific sonographic features for FTCs. Sonography could play a role in differentiating follicular thyroid carcinoma from adenoma.

Keywords: follicular thyroid carcinoma, follicular thyroid adenoma, ultrasonography

\section{Introduction}

Thyroid nodules are very common findings and high-resolution sonography, the preferred method for thyroid nodules, ${ }^{1}$ has drastically multiplied the number of incidentally spotted thyroid nodules. Follicular thyroid adenomas (FTAs) are very common thyroid nodules and follicular thyroid carcinomas (FTCs) represent $10-22 \%$ of thyroid carcinomas, second only to papillary thyroid carcinomas (PTCs) (80-90\%). ${ }^{1}$ Consequently, the rise in thyroid nodules parallels the increment in thyroid follicular neoplasms. However, whether fine needle aspiration biopsy or core needle biopsy and fast frozen pathology cannot differentiate between FTA and FTC, ${ }^{2-4}$ because FTC has similar cytological features to FTA and its diagnosis requires the histologic demonstration of capsular or vascular invasion. Accordingly, patients tend to be advised to undergo surgery for evaluation of capsular or vascular invasion. However, when a biopsy specimen of a thyroid 
nodule reveals a follicular neoplasm, approximately $80-90 \%$ of such lesions will be adenomas and $10-20 \%$ will be carcinomas. ${ }^{5-7}$ Moreover, ultrasonographic features of thyroid cancer have been reported, including solid, hypoechoic, irregular or micro-lobulated margin, taller than wide shape and microcalcifications with sensitivity of $26-87 \%$ and specificity of $53-93 \%$, ${ }^{8,9}$ but these ultrasonographic features are representative of papillary carcinomas. ${ }^{10-13}$ Therefore, the purpose of our study is to explore the sonographic features of FTCs and the value of sonography in differentiating FTC from FTA.

\section{Materials and Methods}

\section{Patients}

This study was approved by Medical Ethics Committee of Chinese PLA General Hospital (S2019-178-02) and the requirement for informed consent was waived because the patients' data were evaluated retrospectively and anonymously. All members of our team are committed to confidentiality of patients' data and compliance with the Declaration of Helsinki. We retrospectively identified 35 patients who received a diagnosis of FTC by thorough histopathologic analysis of resected hemi-thyroidectomy or total thyroidectomy specimens from a pathology report database between January 1, 2015 and August 3, 2020. Of them, nine patients were excluded from the study because of unavailable presurgical sonography. Two FTCs were found in two patients; thus, 28 FTCs of 26 patients (20 females and 6 males; mean age, 45.08 years; range, $11-75$ years) were analyzed. A total of 52 control patients (41 females and 11 males; mean age, 48.56 years; range, 25-74 years) with a diagnosis of FTA were selected from a total of 923 surgical cases of FTA during the study period by simple random sampling method in SPSS software, version 21.0. Two FTAs were found in one patient; therefore, 53 FTAs were analyzed.

\section{Sonography}

Thyroid sonography was carried out using a Philips iU22 or iU Elite scanner (Philips Medical Systems, Bothell, WA, USA) or a GE LOGIQ E9 scanner (GE Healthcare, Wauwatosa, WI, USA) or a VINNO 70 scanner (Vinno Technology, Suzhou, Jiangsu, China) and other scanners with an $8.0-15.0 \mathrm{MHz}$ linear transducer. Patients were scanned in the supine position with the head back. The gain, depth, focus, edge enhancement and dynamic range were adjusted to optimize image during the examination. Two sonologists (with more than 5 years of experience in thyroid sonography) conducted a retrospective review of the sonograms without knowledge of clinical information or final diagnoses. Differences in interpretation were solved by consensus. The images were stored in our workstation system and were presented for readers on a picture archiving in an anonymous random way.

The patient's age, sex, and maximum tumor diameter were recorded and analyzed. And patients were classified by age into 2 groups: $<45$ years, $\geq 45$ years. Nodules were also classified by their longest diameter into 2 categories: $\leq 40 \mathrm{~mm},>40 \mathrm{~mm}$.

In the present study, a mode image (Figure 1) was built up to present the halo characteristics of FTC in detail. And we proposed two new sonographic features: an interrupted halo (Figures 1 and 2) and satellite nodule(s) with or without halo ring (Figures 1 and 3) which were added as parts of the halo feature. Complete capsule penetration and/or tumor growth beyond the capsule could result in an interrupted halo and/or satellite nodule(s). Both are direct signs of capsule invasion of tumors; therefore, nodules with these two features can be directly diagnosed as FTC. Regarding irregular shape, it was categorized as other shapes except for round to oval; taller than wide; local irregularity of margin. The latter was proposed and defined as spiculated, jagged or micro-lobulated changes in part(s) of margin (Figure 4), as distinct from these

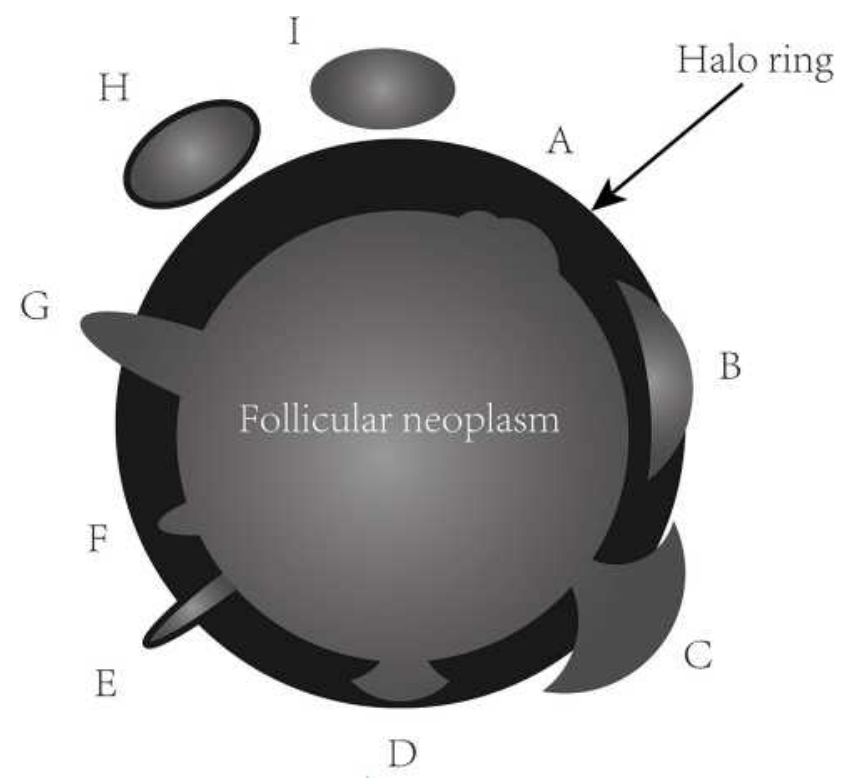

Figure I The thick, irregular and/or interrupted halo ring with or without satellite nodule(s) due to tumor invasion of capsule. The feature of $A$ site can be seen in both follicular adenomas and carcinomas. The features of B, C, E, G, H and I sites can only be observed in follicular carcinomas. $B$ and $C$ features can be presented in different sections of mushroom-shaped tumor bud of follicular carcinoma. Satellite nodules with or without halo ring $(\mathrm{H}$ and $\mathrm{I})$. Tumor bud clothed by a thin halo ring (E). $D$ and $F$ are suspicious features of follicular carcinoma. 


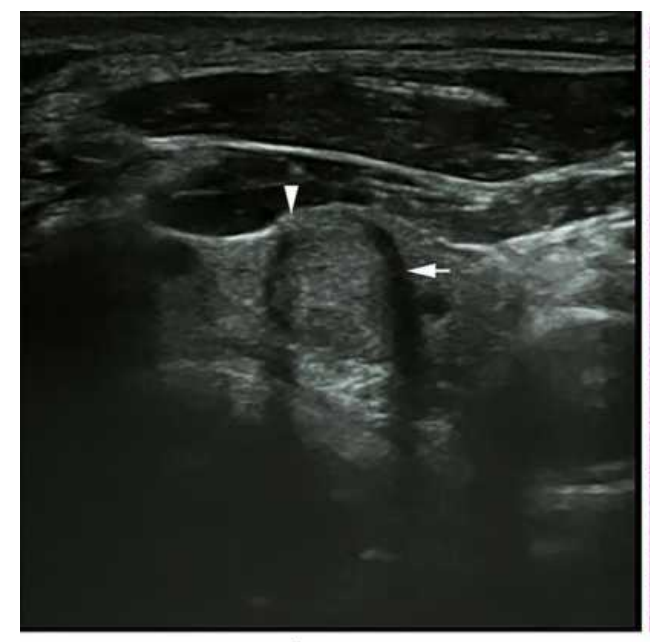

A

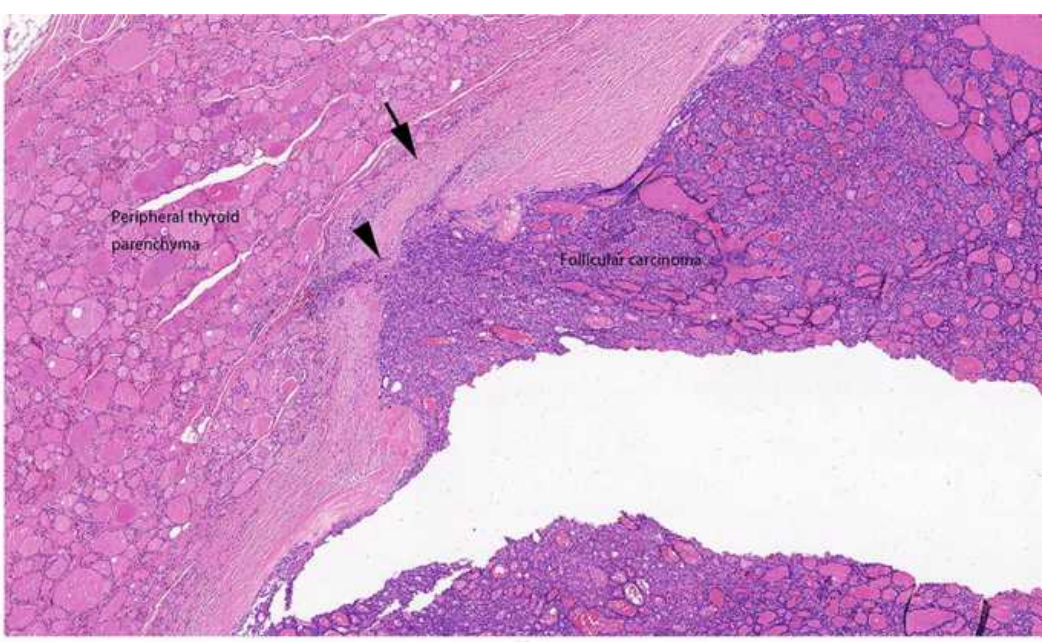

B

Figure 2 Follicular thyroid carcinoma of the left thyroid in a 66-year-old male. (A) Longitudinal sonogram indicates an isoechoic nodule with a thick, irregular, homogeneous and markedly hypoechoic halo (arrow) and the interruption of halo because of tumor invasion (arrowhead). (B) Photomicrograph shows tumor invasion into and through the hyalinized capsule (arrowhead) and the thick and irregular capsule (arrow). Mixed growth pattern can be observed in follicular carcinoma. High cellularity can be observed in the tumor of invasion into the capsule $(\mathrm{H}$ and $\mathrm{E}, \times 40)$.

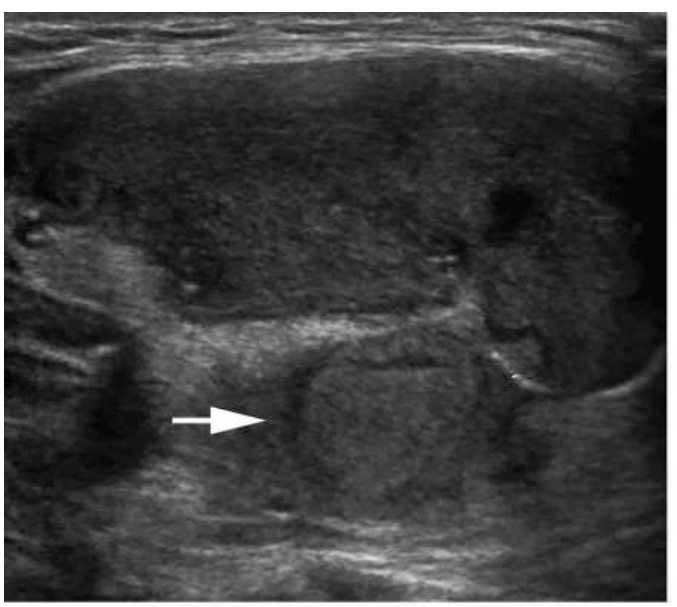

A



B

Figure 3 (A) Follicular thyroid carcinoma of the left thyroid in a 23-year-old female. Longitudinal sonogram shows a hypoechoic nodule with a hypoechoic satellite nodule (arrow). (B) Follicular thyroid carcinoma in a 65-year-old woman. Photomicrograph shows a thick and hyalinized capsule (arrowhead) and a satellite nodule with a secondary limiting fibrous band (arrow) $(\mathrm{H}$ and $\mathrm{E}, \times 100)$.

irregular changes throughout the margin in PTCs. And a feature that appeared as a conglomeration of multiple solid nodules separated by thick and obvious hypoechoic fibrous bands was defined as "cluster of grapes sign" (Figure 5) in our study.

Finally, the following sonographic features were evaluated for each nodule:

- halo (absent; a thin, relatively regular, complete halo (Figure 6A and B); a thick, irregular and/or interrupted halo with or without satellite nodule(s) (Figures 1-3));
- echogenicity (hyperechoic; isoechoic; hypoechoic or markedly hypoechoic);

- echo texture (homogeneous or predominantly homogeneous; heterogeneous);

- shape (round to oval; irregular);

- margin (clear; ill-defined);

- cystic contents (predominantly solid $[\leq 5 \%]$; mixed [5-50\%] or predominantly cystic [>50\%]);

- calcifications (absent; micro-calcifications or macrocalcifications; rim calcifications)

- nodularity (multiple or solitary);

- cluster of grapes sign (present or absent). 


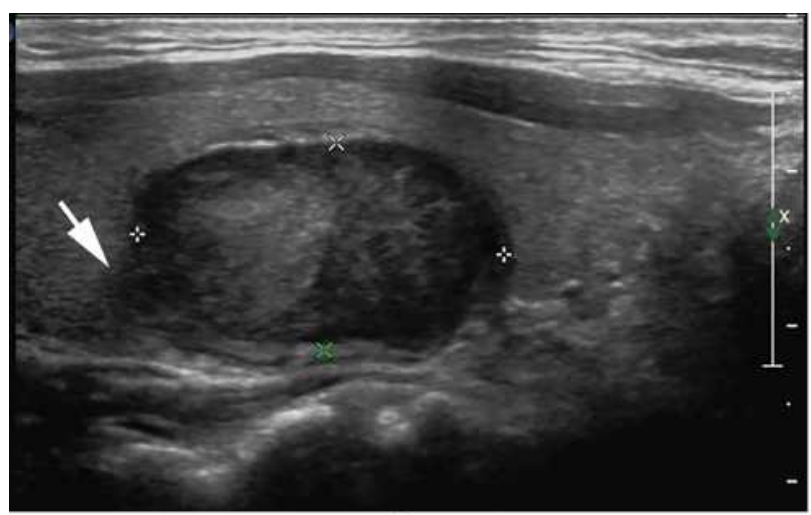

A

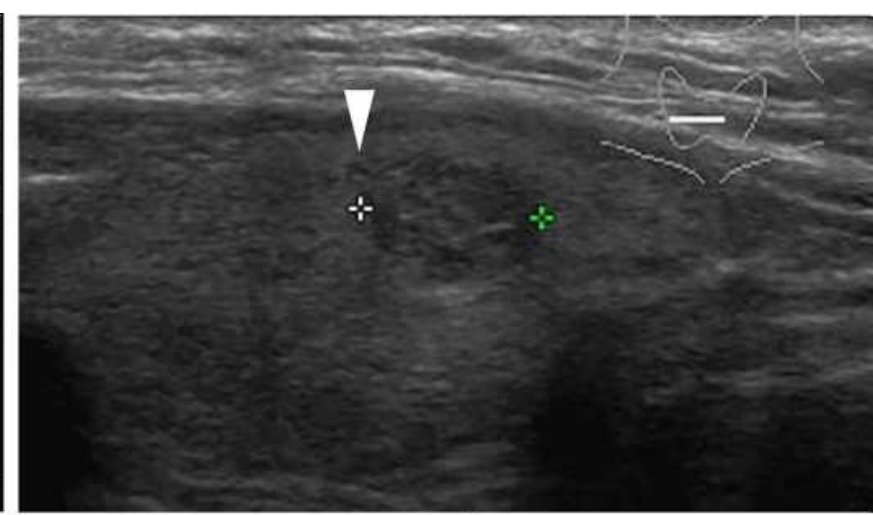

B

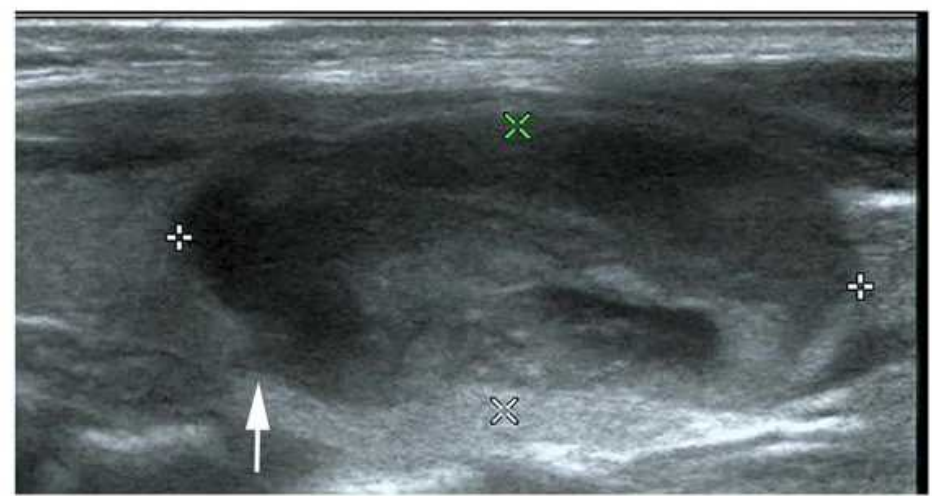

C

Figure 4 (A) Follicular thyroid carcinoma of the right thyroid in a 40-year-old male. Longitudinal sonogram indicates a generally oval nodule but with a lobulated change in the part of margin (arrow). (B) Follicular thyroid carcinoma of the right thyroid in a 40-year-old female. Transverse sonogram indicates a nodule with a spiculated change in the part of margin, deriving from tumor penetration of capsule (arrowhead). (C) Follicular thyroid carcinoma of the left thyroid in a II-year-old female. Longitudinal sonogram shows a generally oval nodule but with a jagged change in the part of margin (arrow).

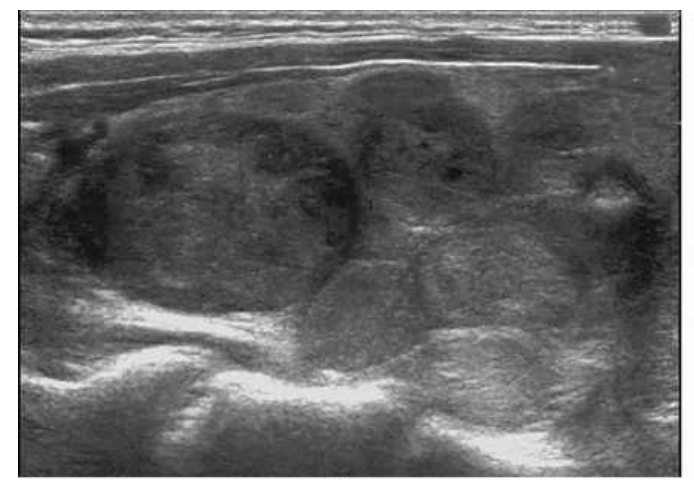

A

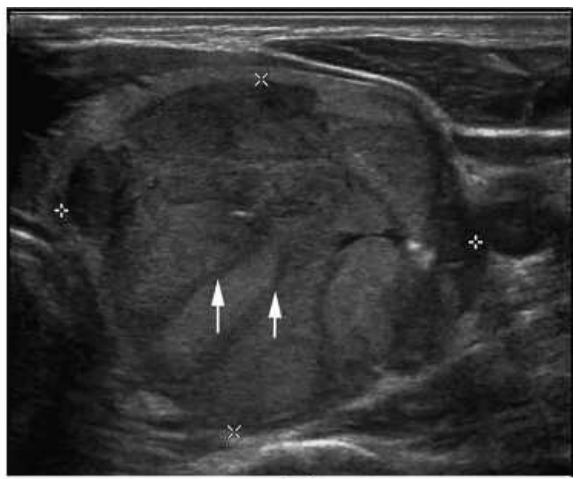

B

Figure 5 (A) Follicular thyroid carcinoma of the left thyroid in a 58-year-old female. Longitudinal sonogram indicates the cluster of grapes sign. (B) Follicular thyroid carcinoma of the left thyroid in a 27 -year-old male. Transverse sonogram shows obvious cluster of grapes sign and the thick and hypoechoic fibrous band (arrow).

\section{Pathology}

The pathological diagnoses and classifications of follicular neoplasm were based on the most recent WHO classification system and were performed by a pathologist of our hospital with more than ten years of experience in the diagnostic histopathology of thyroid tumors. FTA is a benign, encapsulated, non-invasive neoplasm showing evidence of thyroid follicular cell differentiation, without nuclear features of PTC. ${ }^{14}$ FTC is a thyroid malignancy arising from follicular cells in which the diagnostic nuclear features of PTC are absent. The lesions are usually encapsulated and show invasive growth. ${ }^{15}$ The diagnosis of FTC 


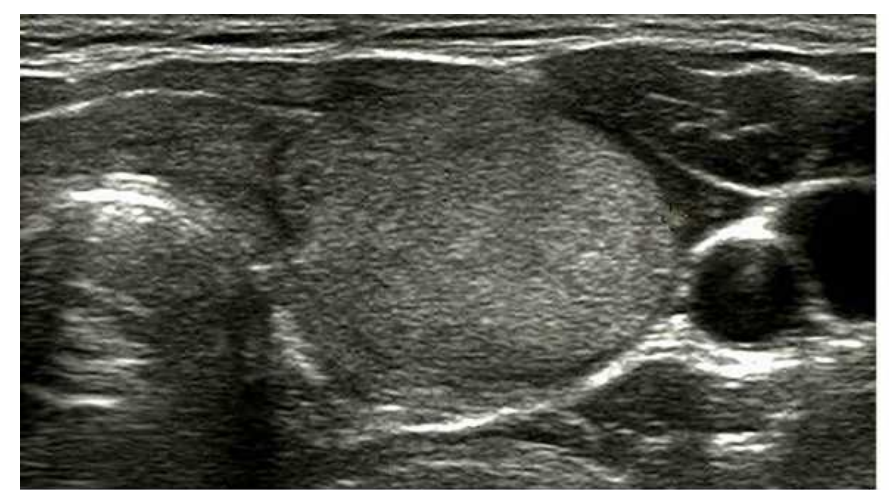

A

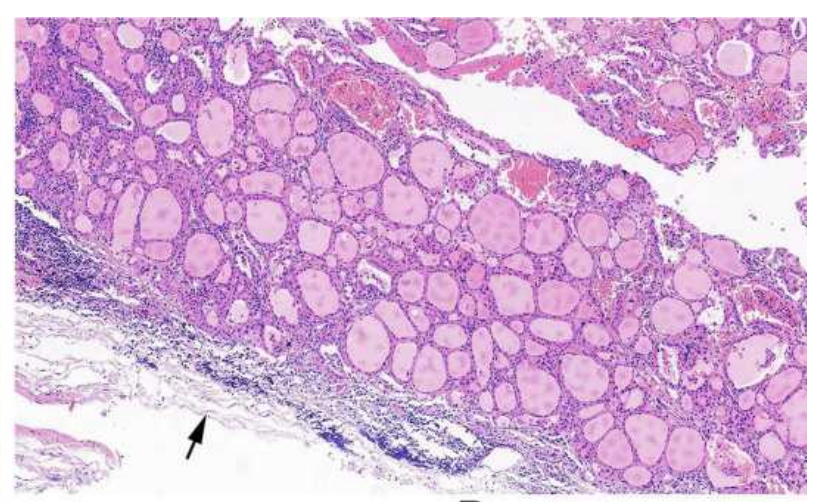

B

Figure 6 Follicular thyroid adenoma of the left thyroid in a 41 -year-old female. (A) Transverse sonogram indicates a nodule with a thin and relatively regular halo. (B) Photomicrograph shows a thin capsule (arrow) and normo-follicular growth pattern can be observed $(\mathrm{H}$ and $\mathrm{E}, \times 100)$.

requires demonstration of capsular and/or vascular invasion. Capsular invasion is defined as the penetration of entire thickness of the tumor capsule. Vascular invasion is invasion into veins, no matter the vessel size. Intravascular tumor cells should be adherent to the vessel walls, either covered by endothelium or in a context of thrombus or fibrin. Invasion must occur in vessels within or beyond the tumor capsule. ${ }^{15}$ FTC is subclassified into three groups: (1) minimally invasive (capsular invasion only), (2) encapsulated angioinvasive, (3) widely invasive. ${ }^{15}$

\section{Statistical Analysis}

The statistical software used was SPSS, version 21.0 (SPSS Inc, Chicago, IL) and STATA, version 15.0 (StataCorp LLC, Texas, USA). Continuous variables (age and maximum diameter) were summarized as mean \pm SD. Categorical variables were summarized as frequencies and percentages. Continuous variables were assessed using unpaired Student $t$ test due to the data Gaussian distribution. The chi-square test or Fisher's exact test was used to evaluate the categorical variables.

Further analysis was carried out using multivariate logistic regression to appraise the relative risks of malignancy. Variables with a $\mathrm{p}$ value of $<0.05$ in univariate analysis were selected in the multivariate logistic regression model. Age and sex were added to the model to check for possible confounding effects. Results were considered significant when odds ratio (OR) was greater than 1.0 and the lower score for the $95 \%$ confidence limit was also greater than 1.0. Then, a nomogram was constructed to better present the results depending on the independent risk factors identified by multivariate logistic regression.
We also determined the usefulness of sonographic features that had statistically significant differences by evaluation of the sensitivity, specificity, overall accuracy, positive predictive value (PPV), and negative predictive value (NPV).

\section{Results}

The clinical and sonographic characteristics of FTAs and FTCs are summarized in Table 1. No significant differences were found in age, sex and mean maximum diameters between FTAs and FTCs.

In terms of halo, a thick, irregular and/or interrupted halo with or without satellite nodule(s) was more common for FTCs (57.1\%). An interrupted halo and satellite nodule(s) with or without halo ring could only be found in FTCs, but the sensitivity was low ( $28.6 \%$ and $7.1 \%$, respectively). The absence of halo was seen in both FTCs and FTAs (25\% and $26.4 \%$, respectively). Cluster of grapes sign was seen in $57.1 \%$ of FTCs compared with in $28.3 \%$ of FTAs $(\mathrm{p}=0.011) .67 .9 \%$ of FTCs were hypoechoic or markedly hypoechoic compared with $28.3 \%$ of FTAs ( $\mathrm{p}=0.001$ ). Hyperechoic nodules were rare in both FTCs and FTAs (7.1\% and $3.8 \%$, respectively). Concerning shape, more than half of FTCs were irregular (57.1\%), while most FTAs were round to oval $(77.4 \% ; \mathrm{p}=0.002)$. Taller than wide shape was rare in FTCs (only 17.9\%). Clear margins were observed in all FTAs and most FTCs (100\% versus $89.3 \%$; $p=0.07)$. Only 3 widely invasive FTCs were found ill-defined. A statistically significant difference $(\mathrm{p}=0.024)$ was found for the echo texture, with heterogeneous echo texture associated with FTCs in $67.9 \%$ of cases and with FTAs in $41.5 \%$ of cases. Rim calcifications were observed in $21.4 \%$ of FTCs and $3.8 \%$ of FTAs $(\mathrm{P}<0.05)$, and internal micro-calcifications 
Table I Clinical and Sonographic Features of Follicular Carcinomas and Adenomas

\begin{tabular}{|c|c|c|c|}
\hline Characteristics & FTC $(n=28)$ & FTA $(n=53)$ & $P$ value \\
\hline $\begin{array}{r}\text { Age, } y^{a} \\
<45\end{array}$ & $\begin{array}{l}45.1 \pm 17.3 \\
13(50)\end{array}$ & $\begin{array}{l}48.6 \pm 12.2 \\
23(44.2)\end{array}$ & $\begin{array}{l}0.365 \\
0.63\end{array}$ \\
\hline $\begin{array}{l}\text { Sex }^{\mathrm{a}} \\
\text { Male }\end{array}$ & $6(23.1)$ & $\mid I(2 \mid .2)$ & 0.846 \\
\hline $\begin{array}{l}\text { Maximum diameters, } \mathrm{mm} \\
\quad \leq 40\end{array}$ & $\begin{array}{l}36.0 \pm 19.6 \\
16(57.1)\end{array}$ & $\begin{array}{l}28.7 \pm I I .5 \\
4 I(77.4)\end{array}$ & $\begin{array}{l}0.07 \\
0.058\end{array}$ \\
\hline $\begin{array}{l}\text { Echogenicity } \\
\text { Hyperechoic } \\
\text { Isoechoic } \\
\text { Hypoechoic or markedly hypoechoic }\end{array}$ & $\begin{array}{l}2(7.1) \\
7(25) \\
19(67.9)\end{array}$ & $\begin{array}{l}2(3.8) \\
36(67.9) \\
15(28.3)\end{array}$ & 0.001 \\
\hline $\begin{array}{l}\text { Echo texture } \\
\text { Homogeneous or predominantly homogeneous } \\
\text { Heterogeneous }\end{array}$ & $\begin{array}{l}9(32.1) \\
19(67.9)\end{array}$ & $\begin{array}{l}3 I(58.5) \\
22(4 I .5)\end{array}$ & 0.024 \\
\hline $\begin{array}{l}\text { Cluster of grapes sign } \\
\text { Present }\end{array}$ & $16(57.1)$ & I5(28.3) & 0.011 \\
\hline $\begin{array}{l}\text { Halo } \\
\text { Absent } \\
\text { A thin, relatively regular, complete halo } \\
\text { A thick, irregular and/or interrupted halo with or without satellite nodule(s) } \\
\text { An interrupted halo } \\
\text { Satellite nodule(s) with or without halo }\end{array}$ & $\begin{array}{l}7(25) \\
5(17.9) \\
16(57.1) \\
8(28.6) \\
2(7.1)\end{array}$ & $\begin{array}{l}14(26.4) \\
27(51.0) \\
12(22.6) \\
0(0) \\
0(0)\end{array}$ & 0.003 \\
\hline $\begin{array}{l}\text { Shape } \\
\text { Round to oval } \\
\text { Irregular }\end{array}$ & $\begin{array}{l}12(42.9) \\
16(57.1)\end{array}$ & $\begin{array}{l}4 \mid(77.4) \\
12(22.6)\end{array}$ & 0.002 \\
\hline $\begin{array}{l}\text { Margin } \\
\text { Clear } \\
\text { III-defined }\end{array}$ & $\begin{array}{l}25(89.3) \\
3(10.7)\end{array}$ & $\begin{array}{l}53(100) \\
0(0)\end{array}$ & 0.07 \\
\hline $\begin{array}{l}\text { Cystic contents } \\
\text { Predominantly solid }(\leq 5 \%) \\
\text { Mix }(5-50 \%) \text { or predominantly cystic }(>50 \%)\end{array}$ & $\begin{array}{l}25(89.3) \\
3(10.7)\end{array}$ & $\begin{array}{l}32(60.4) \\
21(39.6)\end{array}$ & 0.007 \\
\hline $\begin{array}{l}\text { Calcifications } \\
\text { Absence } \\
\text { Micro- or macro-calcifications } \\
\text { Rim calcifications }\end{array}$ & $\begin{array}{l}12(42.9) \\
10(35.7) \\
6(21.4)\end{array}$ & $\begin{array}{l}43(8 I . I) \\
8(I 5 . I) \\
2(3.8)\end{array}$ & 0.001 \\
\hline Solitary & $8(28.6)$ & $7(13.2)$ & 0.09 \\
\hline
\end{tabular}

Notes: Data are presented as mean \pm SD and number (percent); ${ }^{2} 26$ follicular carcinoma patients with 28 nodules, 52 follicular adenoma patients with 53 nodules. Abbreviations: FTC, follicular thyroid carcinoma; FTA, follicular thyroid adenoma.

or macro-calcifications were found in $35.7 \%$ of FTCs and $15.1 \%$ of FTAs $(\mathrm{P}<0 \quad 0.05)$. As to cystic contents, a predominantly solid pattern was more commonly seen in FTCs than in FTAs $(89.3 \%$ versus $60.4 \%$; $p=0.007)$. The ratio of solitary nodule in FTCs was higher than that in FTAs, but no statistically significant difference was found (28.6\% versus $13.2 \% ; \mathrm{P}=0.09)$.
Independent risk factors for FTCs identified by multivariate logistic regression analysis were as follows: a thick, irregular and/or interrupted halo with or without satellite nodule(s) (OR: 11.48, 95\% confidence interval: 1.37-96.56, $\mathrm{p}=0.025$ ), hypoechoic or marked hypoechoic echogenicity (OR: 6.74, 95\% confidence interval: 1.05-43.30, $\mathrm{p}=0.044)$, a predominantly solid pattern (OR: 17.51, 95\% confidence 


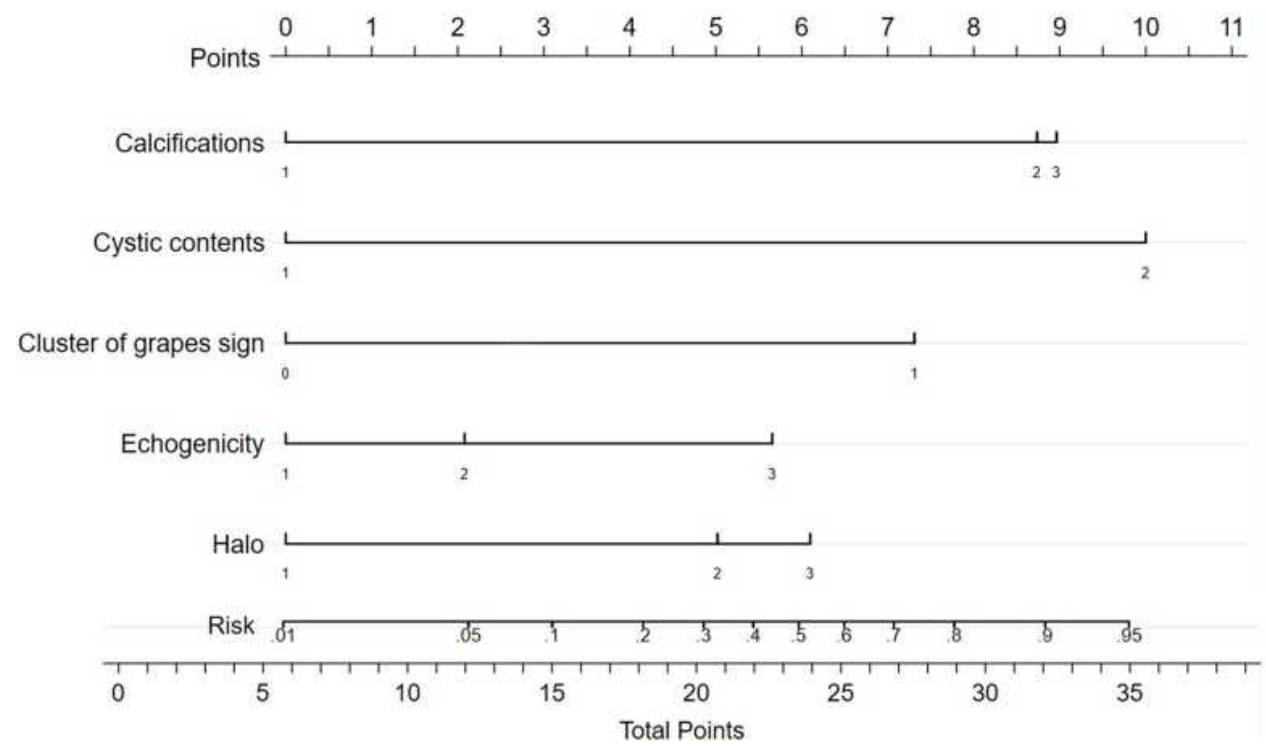

Figure $7 \mathrm{~A}$ nomogram for predicting the malignancy risk of follicular neoplasms. The scores of each variable were added to obtain the total score, and a vertical line was drawn on the total score to obtain the corresponding malignancy risk.

Notes: Calcification (I Absence, 2 Rim calcifications, 3 Micro- or macro-calcifications), Cystic contents (I Mix (5-50\%) or predominantly cystic (>50\%), 2 Predominantly solid ( $\leq 5 \%)$ ), Cluster of grapes sign (0 Absent, I Present), Echogenicity (I Isoechoic, 2 Hyperechoic, 3 Hypoechoic or markedly hypoechoic), Halo (I Athin, relatively regular, complete halo, 2 Absent, 3 Athick, irregular and/or interrupted halo with or without satellite nodule(s)).

interval: $1.78-172.53, \mathrm{p}=0.014)$, cluster of grapes sign (OR: 9.55, 95\% confidence interval: $1.44-63.46, \mathrm{p}=0.02)$, micro- or macro-calcifications (OR: 9.36, 95\% confidence interval: 1.25-70.15, $\mathrm{p}=0.03$ ), rim calcifications (OR: 17.45, 95\% confidence interval: $1.04-292.65, \mathrm{p}=0.047)$. A nomogram (Figure 7) was established to better present the results of multivariate logistic regression analysis.

The sensitivity, specificity, overall accuracy, PPV, and NPV of the sonographic findings that showed a statistically significant difference between FTCs and FTAs are listed in Table 2. It was suggested that when follicular neoplasms with a predominantly solid pattern were diagnosed as FTCs, the sensitivity and NPV were the highest $(89.3 \%$ and $87.5 \%$, respectively), yet the specificity (39.6\%) and PPV (43.9\%) were lower. Still, there was an enhancement in specificity and PPV when a predominantly solid pattern was combined with other statistically significant features. The combination of a predominantly solid pattern and calcifications could attain the highest specificity and PPV (94.3\% and 82.4\%, respectively). A predominantly solid pattern combined with hypoechoic or markedly hypoechoic echogenicity or calcifications can achieve the highest accuracy (79\%).

\section{Discussion}

Our study confirmed that a thick, irregular and/or interrupted halo with or without satellite nodule(s), hypoechoic or markedly hypoechoic echogenicity, a predominantly solid pattern, cluster of grapes sign, micro- or macrocalcifications and rim calcifications were independent risk factors of FTCs. And an interrupted halo and satellite nodule(s) with or without halo ring are specific sonographic features for FTCs.

A halo is defined as a hypoechoic rim surrounding the neoplasm. Its pathological structure is likely to be the fibrous capsule of tumor. FTCs are typically surrounded by a thick and irregular capsule, ${ }^{15}$ while FTAs are generally surrounded by a thin or moderately thick capsule. ${ }^{14}$ Most pathologists require that the whole thickness of tumor capsule needs to be penetrated by the neoplastic cells in the diagnosis of FTCs. ${ }^{15}$ The following are thought to be the entire penetration of tumor capsule: (1) Tumor bud has invaded beyond the outer contour of the capsule; (2) Tumor bud is still clothed by thin (probably new) fibrous capsule, but it has already extended beyond an imaginary line drawn through outer contour of capsule; (3) Satellite nodule with cellularity and cytoarchitectural features identical to those of the main tumor; (4) Mushroom-shaped tumor bud has totally transgressed fibrous capsule. ${ }^{16}$ The suspicious signs of FTCs are as follows: (1) Sharp tumor bud has invaded into but not through the capsule; (2) Mushroom-shaped tumor bud within, but not through, the capsule. ${ }^{16}$ Therefore, a thin, relatively regular, complete halo can generally be observed in FTAs, while a thick, irregular and/or interrupted halo 
Table 2 Sensitivity, Specificity, Overall Accuracy, PPV, and NPV of Sonographic Features for Distinguishing Follicular Carcinomas from Adenomas

\begin{tabular}{|c|c|c|c|c|c|}
\hline Features & Sensitivity & Specificity & Accuracy & PPV & NPV \\
\hline Irregular shape & 57.1 & 77.4 & 70.4 & 57.1 & 77.4 \\
\hline A thick, irregular and/or interrupted halo with or without satellite nodule(s) & 57.1 & 77.4 & 70.4 & 57.1 & 77.4 \\
\hline Hypoechoic or markedly hypoechoic & 67.9 & 71.7 & 70.4 & 55.9 & 80.9 \\
\hline Heterogeneous echo texture & 67.9 & 58.5 & 61.7 & 46.3 & 77.5 \\
\hline Cluster of grapes sign & 57.1 & 71.7 & 66.7 & 51.6 & 76.0 \\
\hline Predominantly solid & 89.3 & 39.6 & 56.8 & 43.9 & 87.5 \\
\hline Calcifications & 57.1 & 81.1 & 72.8 & 61.5 & 78.2 \\
\hline Predominantly solid + irregular shape & 53.6 & 88.7 & 76.5 & 71.4 & 78.3 \\
\hline $\begin{array}{l}\text { Predominantly solid + a thick, irregular and/or interrupted halo with or without } \\
\text { satellite nodule(s) }\end{array}$ & 57.1 & 86.8 & 76.5 & 69.6 & 79.3 \\
\hline Predominantly solid + hypoechoic or markedly hypoechoic & 64.3 & 86.8 & 79.0 & 72.0 & 82.1 \\
\hline Predominantly solid + heterogeneous echo texture & 60.7 & 77.4 & 71.6 & 58.6 & 78.8 \\
\hline Predominantly solid + cluster of grapes sign & 46.4 & 75.5 & 65.4 & 50.0 & 72.7 \\
\hline Predominantly solid + calcifications & 50.0 & 94.3 & 79.0 & 82.4 & 78.1 \\
\hline
\end{tabular}

Note: Data are presented as number (percent).

Abbreviations: PPV, positive predictive value; NPV, negative predictive value.

with or without a satellite nodule(s) can typically be found in FTCs. In sonogram, both an interrupted halo and a satellite nodule(s) with or without halo were direct and significant signs of FTCs and were proposed in our study. An interrupted halo, this significant sonographic characteristic, was confirmed by Kaoru Kobayashi et al 's study ${ }^{17}$ which showed that tumor protrusion on ultrasonography was a strongly suggestive finding of FTC. A meta-analysis by Borowczyk et $\mathrm{al}^{18}$ also suggested that the most crucial characteristic connected with an increased risk of FTC was the tumor protrusion. Our conclusion was also consistent with the studies of Zhang et $\mathrm{al}^{19}$ and Solbiati et $\mathrm{al}^{20}$. What is more, hyaline degeneration can arise in tumor capsule, especially in FTC capsule. Thus, a homogeneous and markedly hypoechoic halo can be seen. Moreover, the reasons of halo absence were also investigated. Extremely thin capsule in FTAs, identical echo intensity between hypoechoic tumor tissue and capsule, wide infiltration of capsule in widely invasive FTCs could account for this manifestation.

Cluster of grapes sign was more common and obvious in FTCs. This finding is well associated with the multi- nodular growth pattern which is more frequently seen in FTCs. This feature was named "tubercle in nodule" by Kuo et $\mathrm{al}^{21}$. Our result was also in accord with the study of Shin et $\mathrm{al}^{22}$.

The thick halo and obvious cluster of grapes sign may be associated with stromal reaction of tumors. It can be hypothesized that more active stromal reaction in FTCs may result in thicker capsule and fibrous bands within the nodule. More active stromal reaction may be a protection factor, but the mechanism needs to be further explored.

It has been reported that hypoechoic or markedly hypoechoic echogenicity was more frequently seen in FTCs than in FTAs. $^{23,24}$ This conclusion was validated in our study, although some studies ${ }^{13,25}$ found that isoechoic echogenicity was more common in FTCs. FTCs and FTAs have similar architectural growth patterns: normo-follicular, macrofollicular, micro-follicular, solid/trabecular and other patterns. ${ }^{15}$ However, normo-follicular/macro-follicular growth patterns are more commonly seen in FTAs, while solid/trabecular and micro-follicular growth patterns are more frequently found in FTCs. The echogenicity might intimately correlate with tumor growth patterns (Figure 8). The tumor with a solid/ 


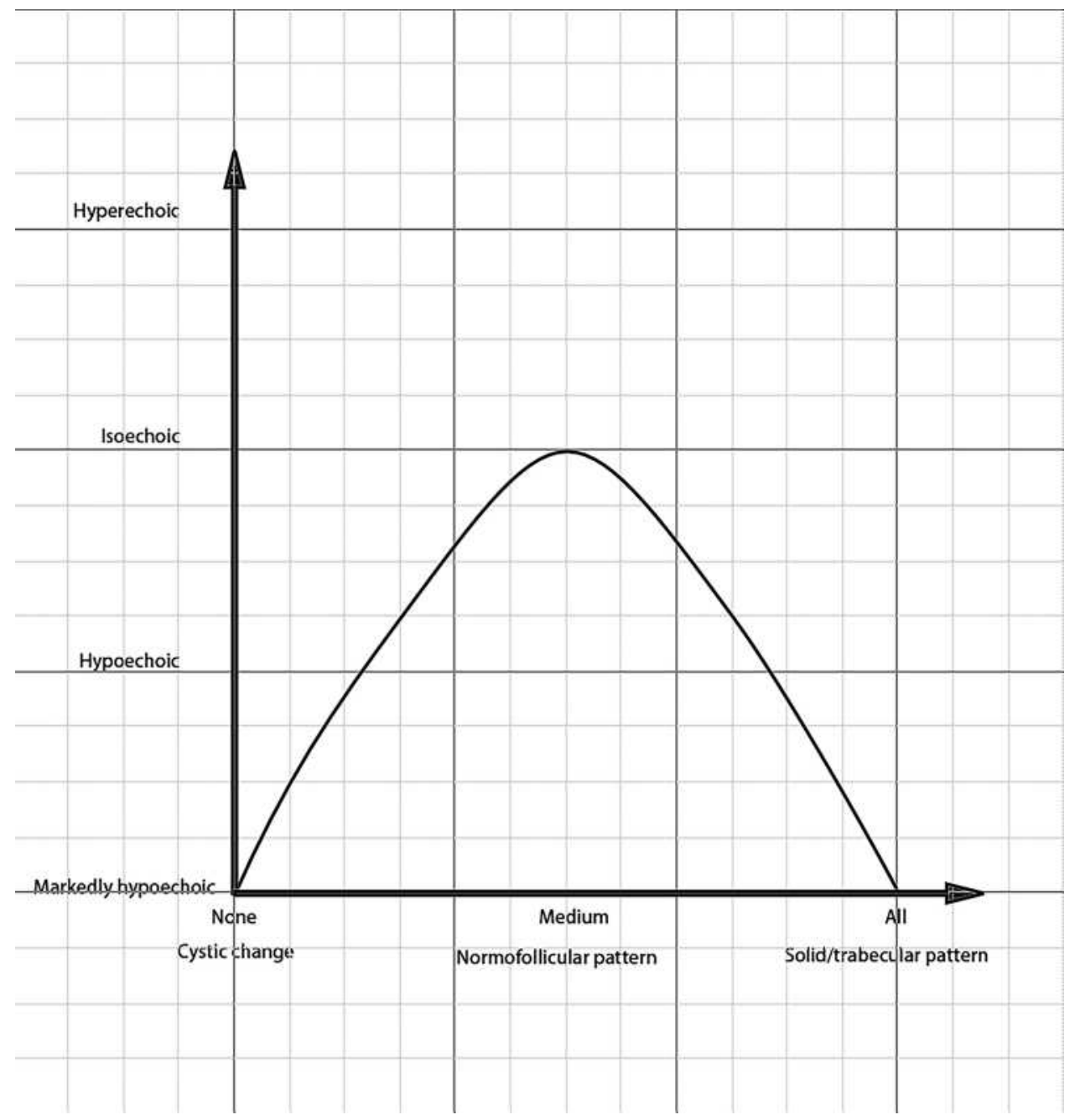

Figure 8 The correlation between echo intensity and tumor growth patterns. The horizontal axis represents cells proportion in cells and colloid of follicular neoplasms, namely cellularity, the vertical axis represents echo intensity.

trabecular growth pattern tends to present markedly hypoechoic intensity, whereas the tumor with macro-follicular and micro-follicular growth patterns tends to show hypoechoic intensity. The tumor with a normo-follicular pattern has a similar echogenic intensity to peripheral thyroid parenchyma, namely iso-echo. Additionally, tumor necrosis, hemorrhage or both in FTCs could also contribute to the finding.

The predictive value of irregular shape is recognized in non-FTCs, but its value in FTCs is unclear and not yet explored. We discovered that irregular shape was more frequently found in FTCs. But irregular shape in FTCs tends to present local irregularity of margin, namely spiculated, jagged or micro-lobulated changes in part(s) of margin, while the above changes are common throughout the margin in PTCs. We hypothesized that FTC is usually an encapsulated lesion, but active hyperplasia of regional cells and/or complete penetration of tumor capsule and consequent tumor growth beyond the capsule can pose local irregularity of margin. On the contrary, PTC is not usually encapsulated; thus, tumor invasion growth can result in common irregular changes along the margin. Liu et $\mathrm{al}^{26}$ and Lai et $\mathrm{al}^{27}$ drew the identical conclusion that lobulated or irregular margin was the significant feature on ultrasound for FTCs. Besides, we found that "taller than wide" in FTCs was rare (17.9\% in our study and only $4-11 \%$ in the study of Cordes et al ${ }^{11}$ ). By contrast, due to capsule confinement and relatively even tumor growth, FTA is generally round to oval with smooth margin.

An ill-defined margin is a significant characteristic of thyroid carcinomas, yet there was no significant difference between FTCs and FTAs in our study. Most FTCs and all FTAs have clear margins. It can be explained that FTA is encapsulated and FTC is usually encapsulated. Even if when neoplastic cells/follicles in FTCs penetrate the 
adjacent thyroid parenchyma, a stromal reaction forming a secondary limiting fibrous band is a common finding. ${ }^{15}$

Many studies ${ }^{12,28}$ indicated that no statistical association was observed with echo texture and the likelihood of FTCs. However, we found that heterogeneous echo texture was more commonly seen in FTCs. It can be hypothesized that mixed growth pattern is more common in FTCs. In addition, tissue necrosis, hemorrhage and interstitial fibrosis in FTCs could be responsible for this finding.

There is a close correlation between calcifications and an increased risk of thyroid malignancy. ${ }^{29}$ This conclusion was demonstrated in our study. Compared with the classic appearance of micro-calcifications in PTCs, the patterns of microcalcifications or macro-calcifications in FTCs showed an inclination to be small in quantity and scattered. It can be hypothesized that these calcifications are secondary to tissue necrosis, hemorrhage, or both (namely dystrophic calcifications) which are more common in FTCs, whereas microcalcifications in PTCs are deemed to originate from the psammoma bodies. ${ }^{30,31}$ In addition, rim calcifications may be a valuable feature of FTCs and 6 of 8 rim calcifications were found in FTCs in our study, although rim or eggshell calcification of thyroid nodules has always been suggested as a sign of benignancy. It can be explained that calcifications can occur in the thick and/or hyalinized capsule of FTCs and be distributed along it. ${ }^{15}$

It has been suggested that FTCs rarely become cystic. $^{32,33}$ This conclusion was confirmed in our study. We speculate that rapid proliferation of malignant cells, an increased attachment between malignant cells and capsule, capsule transgression of tumor as well as thickened capsule restriction are combined to result in this finding.

The diagnostic values of age, sex, size and solitary nodule are controversial. ${ }^{19,23,24,34}$ We failed to find any significant differences in age, sex, the size and solitary nodule in our study.

Our study had many limitations. We cannot assess the sonographic features in real time due to the retrospective study design, thereby likely to omit some prominent information. Moreover, owing to the lower incidence of FTCs, the number of patients with FTCs in our study was not large, thus resulting in an incomprehensive evaluation of patients' clinical and sonographic features. Considering that retrospective interpretation of static color Doppler sonograms is not reliable, our study did not include color Doppler finding as a parameter. Further prospective study is needed to solve these problems. And we only investigated FTCs and FTAs, parts of the encapsulated follicular-patterned thyroid tumors, other pathological types have not yet been explored and we will proceed with a series of study in this area. Additionally, the application of machine learning in differentiating FTCs from FTAs using ultrasonography has been reported ${ }^{35}$ and may be a potential research field.

\section{Conclusions}

In conclusion, our study demonstrated that a thick, irregular and/or interrupted halo with or without satellite nodule(s), hypoechoic or markedly hypoechoic echogenicity, a predominantly solid pattern, cluster of grapes sign, microor macro-calcifications and rim calcifications were independent risk factors of FTCs. And an interrupted halo and satellite nodule(s) with or without halo ring were specific sonographic features for FTCs. The differentiation between FTC and FTA is very difficult, because FTC has similar cytological features to FTA and its diagnosis requires the histologic demonstration of capsular or vascular invasion. High-frequency sonography could present dynamic and high-resolution sonograms of thyroid nodules. Combined with some sonographic features, sonography could play a role in differentiating FTCs from FTAs.

\section{Abbreviations}

FTA, follicular thyroid adenoma; FTC, follicular thyroid carcinoma; PTC, papillary thyroid carcinoma.

\section{Ethics Approval and Informed Consent}

This study was approved by Medical Ethics Committee of Chinese PLA General Hospital (S2019-178-02) and the requirement for informed consent was waived because the patients' data were evaluated retrospectively and anonymously. All members of our team are committed to confidentiality of patients' data and compliance with the Declaration of Helsinki.

\section{Acknowledgments}

The authors thank all team members and colleagues in the Department of Ultrasound, the First Medical Center, Chinese PLA General Hospital for their helpful cooperation and all the participants for their patience and support.

\section{Funding}

This work was supported by grants from the National Natural Science Foundation of China (81901746 to Y.Z.) and grants from Beijing Municipal Science and Technology Commission (Z181100001718017 to Y.L.). 


\section{Disclosure}

The authors report no conflicts of interest in this work.

\section{References}

1. Haugen BR, Alexander EK, Bible KC, et al. 2015 American Thyroid Association Management Guidelines for Adult Patients with Thyroid Nodules and Differentiated Thyroid Cancer: the American Thyroid Association Guidelines Task Force on Thyroid Nodules and Differentiated Thyroid Cancer. Thyroid. 2016;26 (1):1-133.

2. Liu X, Medici M, Kwong N, et al. Bethesda categorization of thyroid nodule cytology and prediction of thyroid cancer type and prognosis. Thyroid. 2016;26(2):256-261. doi:10.1089/thy.2015.0376

3. Romero-Rojas AE, Diaz-Perez JA, Mastrodimos M, Chinchilla SI. Follicular thyroid carcinoma with signet ring cell morphology: fine-needle aspiration cytology, histopathology, and immunohistochemistry. Endocr Pathol. 2013;24(4):239-245. doi:10.1007/s12022-013-9271-x

4. Sugino K, Ito K, Nagahama M, et al. Diagnostic accuracy of fine needle aspiration biopsy cytology and ultrasonography in patients with thyroid nodules diagnosed as benign or indeterminate before thyroidectomy. Endocr J. 2013;60(3):375-382. doi:10.1507/endocrj. EJ12-0338

5. Stolf BS, Santos MM, Simao DF, et al. Class distinction between follicular adenomas and follicular carcinomas of the thyroid gland on the basis of their signature expression. Cancer. 2006;106 (9):1891-1900. doi:10.1002/cncr.21826

6. Smith J, Cheifetz RE, Schneidereit N, Berean K, Thomson T. Can cytology accurately predict benign follicular nodules? Am J Surg. 2005;189(5):592-595; discussion 595. doi:10.1016/j.amjsurg.20 05.01 .028

7. Carpi A, Nicolini A, Gross MD, et al. Controversies in diagnostic approaches to the indeterminate follicular thyroid nodule. Biomed Pharmacother. 2005;59(9):517-520. doi:10.1016/j.biopha.20 05.04.003

8. Brito JP, Gionfriddo MR, Al Nofal A, et al. The accuracy of thyroid nodule ultrasound to predict thyroid cancer: systematic review and meta-analysis. J Clin Endocrinol Metab. 2014;99(4):1253-1263. doi:10.1210/jc.2013-2928

9. Nam SY, Shin JH, Han BK, et al. Preoperative ultrasonographic features of papillary thyroid carcinoma predict biological behavior $J$ Clin Endocrinol Metab. 2013;98(4):1476-1482. doi:10.1210/ jc.2012-4072

10. Yoon JH, Lee HS, Kim EK, Moon HJ, Kwak JY. Malignancy risk stratification of thyroid nodules: comparison between the thyroid imaging reporting and data system and the 2014 American Thyroid Association Management Guidelines. Radiology. 2016;278 (3):917-924. doi:10.1148/radiol.2015150056

11. Cordes M, Kondrat P, Uder M, Kuwert T, Sasiadek M. Differential diagnostic ultrasound criteria of papillary and follicular carcinomas: a multivariate analysis. RoFo Fortschritte auf dem Gebiete der Rontgenstrahlen und der Nuklearmedizin. 2014;186(5):489-495. doi:10.1055/s-0034-1366282

12. Koike E, Noguchi S, Yamashita H, et al. Ultrasonographic characteristics of thyroid nodules: prediction of malignancy. Archives Surg. 2001;136(3):334-337. doi:10.1001/archsurg.136.3.334

13. Jeh SK, Jung SL, Kim BS, Lee YS. Evaluating the degree of conformity of papillary carcinoma and follicular carcinoma to the reported ultrasonographic findings of malignant thyroid tumor. Korean J Radiol. 2007;8(3):192-197. doi:10.3348/kjr.2007.8.3.192

14. Nikiforov YE, Baloch ZW, Belge G, Chan JKC. Follicular adenoma. In: Lloyd RV, Osamura RY, Kloppel G, Rosai J, editors. WHO Classification of Tumors of Endocrine Organs. 4th ed. Lyon: IARC; 2017:69.
15. LiVolsi V, Abdulkader NI, Baloch ZW, Bartolazzi A. Follicular thyroid carcinoma. In: Lloyd RV, Osamura RY, Kloppel G, Rosai J, editors. WHO Classification of Tumors of Endocrine Organs. 4th ed. Lyon: IARC; 2017:92-93.

16. Chan JKC. The thyroid gland. In: Fletcher CDM. editor. Diagnostic Histopathology of Tumors. Vol. 2. 4th edn. Philadelphia: Elsevier Saunders; 2013:1204.

17. Kobayashi K, Hirokawa M, Yabuta T, et al. Tumor protrusion with intensive blood signals on ultrasonography is a strongly suggestive finding of follicular thyroid carcinoma. Med Ultrason. 2016;18 (1):25-29. doi:10.11152/mu.2013.2066.181.kok

18. Borowczyk M, Woliński K, Więckowska B, et al. Sonographic features differentiating follicular thyroid cancer from follicular Adenoma-A meta-analysis. Cancers. 2021;13(5):938. doi:10.3390/ cancers 13050938

19. Zhang JZ, Hu B. Sonographic features of thyroid follicular carcinoma in comparison with thyroid follicular adenoma. J Ultrasound Med. 2014;33(2):221-227. doi:10.7863/ultra.33.2.221

20. Solbiati L, Osti V, Cova L, Tonolini M. Ultrasound of thyroid, parathyroid glands and neck lymph nodes. Eur Radiol. 2001;11 (12):2411-2424. doi:10.1007/s00330-001-1163-7

21. Kuo TC, Wu MH, Chen KY, Hsieh MS, Chen A, Chen CN. Ultrasonographic features for differentiating follicular thyroid carcinoma and follicular adenoma. Asian $j$ Surg. 2020;43(1):339-346. doi:10.1016/j.asjsur.2019.04.016

22. Shin JH, Han BK, Ko EY, Oh YL, Kim JH. Differentiation of widely invasive and minimally invasive follicular thyroid carcinoma with sonography. Eur J Radiol. 2010;74(3):453-457. doi:10.1016/j. ejrad.2009.03.019

23. Sillery JC, Reading CC, Charboneau JW, Henrichsen TL, Hay ID, Mandrekar JN. Thyroid follicular carcinoma: sonographic features of 50 cases. AJR Am J Roentgenol. 2010;194(1):44-54. doi:10.2214/ AJR.09.3195

24. Seo HS, Lee DH, Park SH, Min HS, Na DG. Thyroid follicular neoplasms: can sonography distinguish between adenomas and carcinomas? J Clin Ultrasound. 2009;37(9):493-500. doi:10.1002/ jcu. 20625

25. Castellana M, Piccardo A, Virili C, et al. Can ultrasound systems for risk stratification of thyroid nodules identify follicular carcinoma? Cancer Cytopathol. 2020;128(4):250-259. doi:10.1002/cncy.22235

26. Liu BJ, Zhang YF, Zhao CK, Wang HX, Li MX, Xu HX. Conventional ultrasound characteristics, TI-RADS category and shear wave speed measurement between follicular adenoma and follicular thyroid carcinoma. Clin Hemorheol Microcirc. 2020;75 (3):291-301. doi:10.3233/CH-190750

27. Lai X, Jiang Y, Zhang B, et al. Preoperative sonographic features of follicular thyroid carcinoma predict biological behavior: a retrospective study. Medicine. 2018;97(41):e12814. doi:10.1097/ MD.0000000000012814

28. Rago T, Di Coscio G, Basolo F, et al. Combined clinical, thyroid ultrasound and cytological features help to predict thyroid malignancy in follicular and Hürthle cell thyroid lesions: results from a series of 505 consecutive patients. Clin Endocrinol (Oxf). 2007;66 (1):13-20. doi:10.1111/j.1365-2265.2006.02677.x

29. Moon WJ, Jung SL, Lee JH, et al. Benign and malignant thyroid nodules: US differentiation-multicenter retrospective study. Radiology. 2008;247(3):762-770. doi:10.1148/radiol.2473070944

30. Frates MC, Benson CB, Charboneau JW, et al. Management of thyroid nodules detected at US: society of Radiologists in Ultrasound consensus conference statement. Radiology. 2005;237 (3):794-800. doi:10.1148/radiol.2373050220

31. Kim EK, Park CS, Chung WY, et al. New sonographic criteria for recommending fine-needle aspiration biopsy of nonpalpable solid nodules of the thyroid. AJR Am J Roentgenol. 2002;178 (3):687-691. doi:10.2214/ajr.178.3.1780687 
32. Weber AL, Randolph G, Aksoy FG. The thyroid and parathyroid glands. CT and MR imaging and correlation with pathology and clinical findings. Radiol Clin North Am. 2000;38(5):1105-1129. doi:10.1016/S0033-8389(05)70224-4

33. Pompili G, Tresoldi S, Primolevo A, De Pasquale L, Di Leo G, Cornalba G. Management of thyroid follicular proliferation: an ultrasound-based malignancy score to opt for surgical or conservative treatment. Ultrasound Med Biol. 2013;39(8):1350-1355. doi:10.1016/j.ultrasmedbio.2013.02.464
34. Paramo JC, Mesko T. Age, tumor size, and in-office ultrasonography are predictive parameters of malignancy in follicular neoplasms of the thyroid. Endocrine Pract. 2008;14(4):447-451. doi:10.4158/ EP.14.4.447

35. Shin I, Kim YJ, Han K, et al. Application of machine learning to ultrasound images to differentiate follicular neoplasms of the thyroid gland. Ultrasonography (Seoul, Korea). 2020;39(3):257-265. doi:10.14366/usg.19069

\section{Publish your work in this journal}

Cancer Management and Research is an international, peer-reviewed open access journal focusing on cancer research and the optimal use of preventative and integrated treatment interventions to achieve improved outcomes, enhanced survival and quality of life for the cancer patient.
The manuscript management system is completely online and includes a very quick and fair peer-review system, which is all easy to use. Visit http://www.dovepress.com/testimonials.php to read real quotes from published authors. 